\title{
A clinicopathologic prediction model for postoperative recurrence in stage Ia non-small cell lung cancer
}

\author{
Yang Zhang, MD, ${ }^{\mathrm{a}, \mathrm{b}}$ Yihua Sun, MD, ${ }^{\mathrm{a}, \mathrm{b}}$ Jiaqing Xiang, MD, ${ }^{\mathrm{a}, \mathrm{b}}$ Yawei Zhang, MD, ${ }^{\mathrm{a}, \mathrm{b}}$ Hong Hu, MD, ${ }^{\mathrm{a}, \mathrm{b}}$ and \\ Haiquan Chen, $\mathrm{MD}^{\mathrm{a}, \mathrm{b}}$
}

\begin{abstract}
Objective: Controversy remains over the appropriate postoperative management for patients with stage Ia non-small cell lung cancer who underwent complete surgical resection as a result of a heterogeneous prognosis. We aimed to identify the predictive factors for recurrence in these patients to aid in the decision making.
\end{abstract}

\begin{abstract}
Methods: We reviewed 344 patients with stage Ia non-small cell lung cancer to analyze the associations between recurrence-free survival and the following clinicopathologic variables: age, gender, smoking history, family history, preoperative serum carcinoembryonic antigen level, type of surgical resection, tumor location, tumor histology, lymphovascular invasion, tumor differentiation, and pathologic T status.

Results: Cox multivariate survival analysis revealed that central tumor location $(P=.019)$, stage T1b $(P=.006)$, high histologic grade (including large cell carcinoma, solid predominant, micropapillary predominant, and invasive mucinous adenocarcinoma, $P=.007)$, poor differentiation $(P=.022)$, and lymphovascular invasion $(P=.035)$ were independently associated with recurrence-free survival. A nomogram for predicting the probability of 3-year recurrence-free survival was developed using the 5 variables. This model shows good calibration, reasonable discrimination (concordance index $=0.733)$, and small overfitting $(2.6 \%)$ demonstrated by bootstrapping.
\end{abstract}

Conclusions: We developed a clinicopathologic prediction model for postoperative recurrence in stage Ia non-small cell lung cancer. This model can help with the selection of appropriate postoperative therapeutic strategies for these patients. (J Thorac Cardiovasc Surg 2014;148:1193-9)

Lung cancer remains the leading cause of cancer-related death worldwide. ${ }^{1}$ Even for patients with pathologic stage Ia non-small cell lung cancer (NSCLC), the 5-year overall survival is only $73 \%,{ }^{2}$ suggesting the presence of prognostic factors other than disease stage.

Surgical resection offers the best curative option for stage Ia NSCLC. However, recurrence after surgery will develop in approximately $20 \%$ of these patients, which is the major cause of death. ${ }^{3,4} \mathrm{Up}$ to now, some clinicopathologic variables have been reported to be associated with survival in early-stage lung cancers, such as tumor size, lymphovascular invasion, preoperative serum carcinoembryonic antigen (CEA) level, gender, and adenocarcinoma histologic subtypes, ${ }^{3,5-8}$ supporting the notion that stage Ia NSCLC is a heterogeneous group regarding prognosis.

\footnotetext{
From the Department of Thoracic Surgery, ${ }^{\text {a }}$ Fudan University Shanghai Cancer Center, Shanghai, China; and Department of Oncology, ${ }^{\mathrm{b}}$ Shanghai Medical College, Fudan University, Shanghai, China.

Disclosures: Authors have nothing to disclose with regard to commercial support.

Received for publication Sept 1, 2013; revisions received Feb 16, 2014; accepted for publication Feb 21, 2014; available ahead of print March 22, 2014.

Address for reprints: Haiquan Chen, MD, Department of Thoracic Surgery, Fudan University Shanghai Cancer Center, 270 Dong-An Rd, Shanghai 200032, China (E-mail: hqchen1@yahoo.com).

$0022-5223 / \$ 36.00$

Copyright (c) 2014 by The American Association for Thoracic Surgery

http://dx.doi.org/10.1016/j.jtcvs.2014.02.064
}

The survival advantage of postoperative adjuvant chemotherapy in stage Ia NSCLC remains controversial and seems to be present only in those with poor prognostic factors, such as larger tumor size and vessel invasion. ${ }^{3,9,10}$ Therefore, stratifying stage Ia NSCLC into different prognostic categories is of crucial importance for appropriate postoperative management.

In this study, we aimed to identify the independent prognostic factors of recurrence-free survival (RFS) specifically in patients with stage Ia NSCLC. A nomogram for 3-year RFS probability was also built to facilitate the selection of high-risk patients.

\section{PATIENTS AND METHODS \\ Patients}

From April 2008 to February 2012, we investigated a series of patients who underwent surgical resection with curative intention at the Department of Thoracic Surgery, Fudan University Shanghai Cancer Hospital, Shanghai, China. Preoperative tests included contrast-enhanced chest computed tomography (CT) scans, cardiopulmonary tests, brain magnetic resonance imaging or brain CT, bone scanning, and abdominal CT or abdominal ultrasonography. The following clinicopathologic data were prospectively collected: age, gender, smoking history, family history of lung cancer, preoperative serum CEA level, time of surgery, type of surgical resection, tumor location, tumor histology, lymphovascular invasion, tumor differentiation, pathologic TNM staging, time to recurrence, and time to last follow-up. Tumors with the center located within the outer one third of the lung on CT scans were considered as peripheral (all of the CT scans were reviewed). Histologic subtypes of lung adenocarcinoma were 


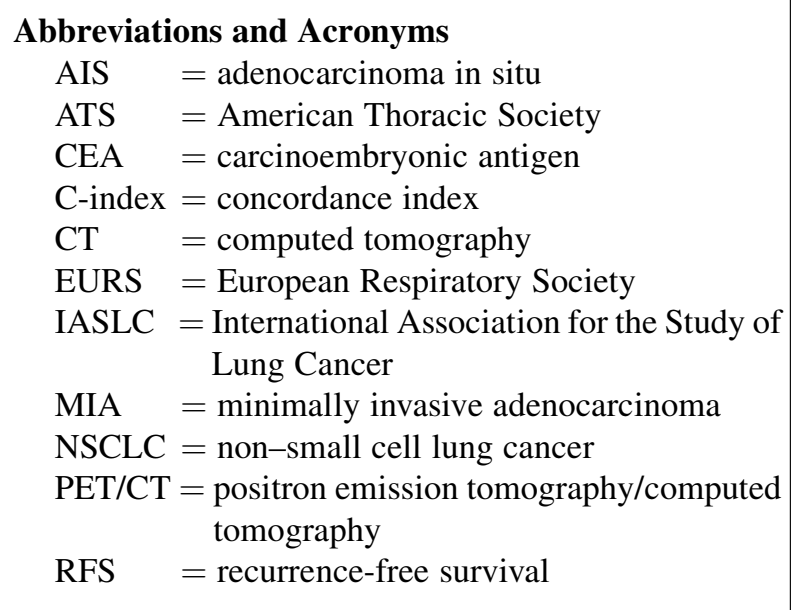

classified in line with the new International Association for the Study of Lung Cancer, American Thoracic Society, and European Respiratory Society (IASLC/ATS/ERS) multidisciplinary lung adenocarcinoma classification. ${ }^{11}$ Lymphovascular invasion was defined as the identification of tumor cells in the lymphatic or blood vessel lumen. Tumor differentiation was determined according to the 2004 World Health Organization classification. ${ }^{12}$ All of the pathologic classifications (including histologic subtypes, lymphovascular invasion, and tumor differentiation) were conducted by 2 qualified pathologists independently, and rare discrepancies were resolved through reexamination of the slides and discussion. TNM stage was classified according to the Seventh Edition of the Lung Cancer Staging system. ${ }^{2}$

Patients were scheduled to be followed up in the clinic every 4 months for the first 3 years after the operation, every 6 months for 3 to 5 years, and every 12 months thereafter. At each follow-up, we routinely performed brain magnetic resonance imaging or brain $\mathrm{CT}$, chest $\mathrm{CT}$ scans, bone scanning, and ultrasonography of the supraclavicular and abdominal regions to detect any evidence of local/regional or distant disease recurrence. Telephone follow-up also was performed as a complement. Patients reporting clinical symptoms during telephone follow-up were encouraged to receive imaging. RFS was defined as the time from the date of surgery to recurrence of lung cancer. Patients without recurrence were censored at the time of last negative follow-up or death without evidence of recurrence. Differentiation between recurrent lung cancer and second primary lung cancer was conducted in line with the criteria reported by Martini and Melamed. ${ }^{13}$ Study inclusion criteria were pathologically confirmed stage Ia (T1a/T1bNOM0) NSCLC and complete resection with systematic lymph node dissection. Exclusion criteria were neoadjuvant chemotherapy or radiotherapy, adjuvant chemotherapy or radiotherapy before disease recurrence, and a history of malignant tumors.

This study was performed in line with the Helsinki Declaration. Our institutional review board approved this study. Because this was a retrospective study, informed consent was waived.

\section{Statistical Analysis}

The RFS distribution was analyzed using the Kaplan-Meier method, and log-rank tests were used for comparisons of RFS between 2 categories in univariate analysis. Variables with a $P$ value of less than .05 were entered into the multivariate survival analysis that was conducted using the Cox proportional hazards regression (forward likelihood ratio model) to identify independent prognostic factors. A nomogram for 3-year RFS probability was built on the basis of the Cox regression model. Independent prognostic factors $(P<.05)$ identified by Cox regression were included in the nomogram.

The discriminative ability of prognostic models was evaluated by Harrell's concordance index (C-index), which is a natural extension of the receiver operating characteristic curve area to survival analysis and ranges from 0.5 (no discrimination) to 1 (perfect discrimination). ${ }^{14}$ The calibration (concordance between predicted and observed probabilities) of the prediction model was assessed by a visual inspection of a calibration plot comparing the predicted and observed 3-year RFS probability of 4 quartiles of patients categorized by nomogram score in each dataset. To internally validate the predictive accuracy of the nomogram, 1000 bootstrap resamples were used to estimate the bias-corrected C-index and the extent of "overfitting." 15

We used SPSS 16.0 (SPSS Inc, Chicago, Ill), Stata 11.1 (StataCorp LP, College Station, Tex), and R 2.10.1 (R Foundation for Statistical Computing, Vienna, Austria) for statistical analysis. All tests were 2 tailed.

\section{RESULTS}

According to the inclusion criteria, 407 patients were identified initially. A total of 52 patients were excluded because of a history of malignant tumors, and 11 patients were excluded because of neoadjuvant chemotherapy (or in combination with neoadjuvant radiotherapy). Finally, 344 patients with NSCLC were included in this study (Table 1). There were 163 women $(47.4 \%)$ and 181 men $(52.6 \%)$ ranging in age from 27 to 83 years (median, 60 years). Lobectomy was performed in 309 patients $(89.8 \%)$, wedge resection was performed in 25 patients $(7.3 \%)$, bi-lobectomy was performed in 8 patients $(2.3 \%)$, and pneumonectomy was performed in 2 patients $(0.6 \%)$. All of the 10 patients receiving bi-lobectomy or pneumonectomy had centrally located tumors. Detailed clinicopathologic characteristics are listed in Table 1.

The median follow-up duration was 31 months. All of the patients completed the follow-up. Disease recurrence was found in 43 patients $(12.5 \%)$, counting as events in the analysis of RFS; locoregional recurrence was found in 19 patients $(44.2 \%)$; and distant recurrence was found in 24 patients $(55.8 \%)$. Histologic confirmation was obtained in 31 patients $(72.1 \%)$, and clinical diagnosis was made for the rest of the patients on the basis of radiologic features. The median time to recurrence in this series of patients was 15 months.

None of the adenocarcinoma in situ (AIS) or minimally invasive adenocarcinoma (MIA) recurred. Disease recurrence seemed to be more likely to occur in patients with the following histologic subtypes of NSCLC: micropapillary predominant adenocarcinoma ( 1 event in 3 patients), solid predominant adenocarcinoma (6 events in 14 patients), invasive mucinous adenocarcinoma ( 2 events in 9 patients), and large cell carcinoma ( 2 events in 4 patients). We classified the tumors with these 4 histologic subtypes as the "high-grade" group. In univariate analysis, the clinicopathologic factors that showed a statistically significant association with worse RFS were male gender $(P=.010)$, ever smokers $(P=.021)$, high preoperative serum CEA 
TABLE 1. Patient characteristics and univariate analysis of predictors of recurrence-free survival in 344 patients with stage Ia non-small cell lung cancer

\begin{tabular}{|c|c|c|}
\hline Variable & Recurrence/no. & $P$ value \\
\hline Gender & & .010 \\
\hline Female & $12 / 163$ & \\
\hline Male & $31 / 181$ & \\
\hline Age (y) & & 275 \\
\hline$>60$ & $24 / 171$ & \\
\hline$\leq 60$ & $19 / 173$ & \\
\hline Smoking history & & . 021 \\
\hline Ever & $25 / 140$ & \\
\hline Never & $18 / 204$ & \\
\hline Family history & & .933 \\
\hline Yes & $3 / 23$ & \\
\hline No & $40 / 321$ & \\
\hline Location & & .003 \\
\hline Central & $11 / 40$ & \\
\hline Peripheral & $32 / 304$ & \\
\hline Lobar distribution & & .865 \\
\hline Upper lobes & $28 / 215$ & \\
\hline Others & $15 / 129$ & \\
\hline CEA (ng/mL) & & .005 \\
\hline$>2.5$ & $26 / 142$ & \\
\hline$\leq 2.5$ & $17 / 202$ & \\
\hline Extent of surgery & & $.760^{*}$ \\
\hline Wedge/segmentectomy & $0 / 25$ & \\
\hline Lobectomy & $41 / 309$ & \\
\hline Bi-lobectomy & $1 / 8$ & \\
\hline Pneumonectomy & $1 / 2$ & \\
\hline Type of surgery & & .068 \\
\hline VATS & $13 / 157$ & \\
\hline Thoracotomy & $30 / 187$ & \\
\hline Tumor size $(\mathrm{cm})$ & & $<.001$ \\
\hline$>2.0$ & $15 / 129$ & \\
\hline$\leq 2.0$ & $28 / 215$ & \\
\hline Histology & & $<.001 \dagger$ \\
\hline Adenocarcinoma & $29 / 261$ & \\
\hline AIS & $0 / 18$ & \\
\hline MIA & $0 / 16$ & \\
\hline Lepidic & $2 / 47$ & \\
\hline Acinar & $16 / 125$ & \\
\hline Papillary & $2 / 29$ & \\
\hline Micropapillary & $1 / 3$ & \\
\hline Solid & $6 / 14$ & \\
\hline IMA & $2 / 9$ & \\
\hline Squamous cell carcinoma & $10 / 65$ & \\
\hline Adenosquamous & $1 / 7$ & \\
\hline Large cell carcinoma & $2 / 4$ & \\
\hline Other NSCLC & $1 / 7$ & \\
\hline Differentiation & & $<.001$ \\
\hline Poor & $21 / 84$ & \\
\hline Moderate/well & $22 / 260$ & \\
\hline Lymphovascular invasion & & $<.001$ \\
\hline Yes & $9 / 16$ & \\
\hline No & $34 / 328$ & \\
\hline
\end{tabular}

$A I S$, Adenocarcinoma in situ; $C E A$, carcinoembryonic antigen; $I M A$, invasive mucinous adenocarcinoma; MIA, minimally invasive adenocarcinoma; NSCLC, non-small cell lung cancer; VATS, video-assisted thoracoscopic surgery. *Wedge, segmentectomy, and lobectomy versus bi-lobectomy and pneumonectomy. †Large cell carcinoma, solid-predominant adenocarcinoma, micropapillary-predominant adenocarcinoma, and invasive mucinous adenocarcinoma versus other histologic subtypes.
TABLE 2. Independent predictors of recurrence-free survival in patients with stage Ia non-small cell lung cancer

\begin{tabular}{llcc}
\hline \multicolumn{1}{c}{ Variable } & \multicolumn{1}{c}{ Category } & HR $(\mathbf{9 5} \% \mathbf{C I})$ & $\boldsymbol{P}$ \\
\hline Location & Central/peripheral & $2.469(1.163-5.242)$ & .019 \\
T stage & T1b/T1a & $2.393(1.277-4.482)$ & .006 \\
Histology & High grade*/others & $3.011(1.356-6.684)$ & .007 \\
Differentiation & Poor/moderate or well & $2.077(1.113-3.878)$ & .022 \\
Lymphovascular & Yes/no & $2.407(1.063-5.448)$ & .035 \\
$\quad$ invasion & & & \\
\hline
\end{tabular}

$C I$, Confidence interval; $H R$, hazard ratio. *High grade includes large cell carcinoma, solid-predominant adenocarcinoma, micropapillary-predominant adenocarcinoma, and invasive mucinous adenocarcinoma.

levels $(P=.005)$, central tumor location $(P=.003)$, stage T1b $(P<.001)$, poor differentiation $(P<.001)$, lymphovascular invasion $(P<.001)$, and high histologic grade $(P<.001)$.

Because no AIS or MIA was reported to recur within 5 years, we excluded the 2 histologic subtypes in Cox multivariate survival analysis, which formed the basis of the prediction nomogram. Central tumor location $(P=.019)$, stage T1b $(P=.006)$, high histologic grade $(P=.007)$, poor differentiation $(P=.022)$, and lymphovascular invasion $(P=.035)$ were independently associated with diseasefree survival (Table 2). On the basis of the Cox model, a nomogram was developed to calculate the 3-year RFS probability (Figure 1$)$. For example, for a $1.5-\mathrm{cm}$ (0 point) peripherally located ( 0 point), poorly differentiated (67 points), solid predominant adenocarcinoma (98 points) without lymphovascular invasion (0 point), the 3 -year RFS probability after surgery is estimated to be between $60 \%$ and $70 \%$ (165 total points).

The calibration plot (Figure 2) showed that the predicted 3-year RFS probabilities closely matched the observed probabilities in each of the 4 risk ranges. The $R^{2}$ value for this model was 0.470 . The $\mathrm{C}$-index was 0.733 for the 5 -predictor prognostic model, whereas using $\mathrm{T}$ stage alone as a prognostic factor achieved only a $\mathrm{C}$-index of 0.630 . The bias-corrected $\mathrm{C}$-index for this nomogram was 0.714 , and the extent of "over-optimism" was small $(2.6 \%)$, indicating that this nomogram holds for future patients. The Kaplan-Meier survival curves stratified by the 5 independent risk factors are shown in Figure 3.

\section{DISCUSSION}

Although previous studies have been carried out to identify clinicopathologic predictors in early-stage lung cancers, ${ }^{3,5-8,16,17}$ this study has several unique aspects. First, we included only patients with pathologic stage Ia who did not receive neoadjuvant chemotherapy or adjuvant therapy before disease recurrence, which allowed the exploration of prognostic factors of RFS without the effect of adjuvant therapy. Second, a comprehensive panel of clinicopathologic variables including adenocarcinoma subtypes 


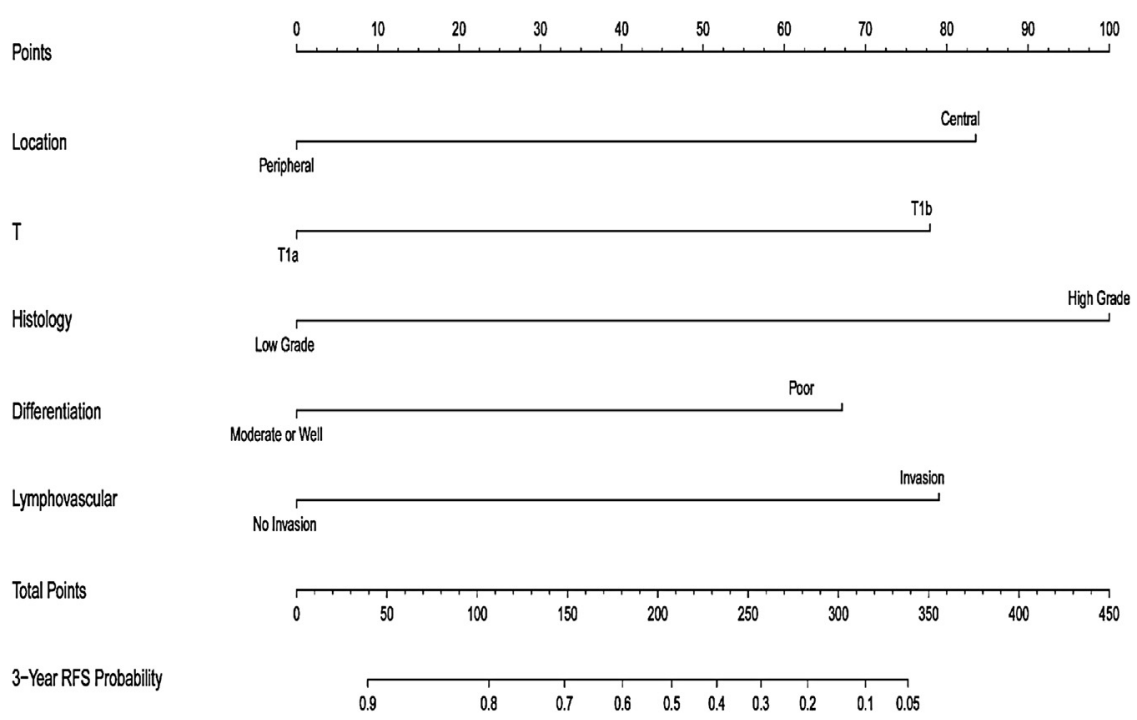

FIGURE 1. Nomogram to predict the probability of 3-year RFS. "High-grade" histology included large cell carcinoma, solid predominant, micropapillary predominant, and invasive mucinous adenocarcinoma. RFS, Recurrence-free survival.

in line with the new IASLC/ATS/ERS classification was collected, and Cox multivariate analysis was performed to identify independent predictors of RFS. We also excluded AIS and MIA in multivariate analysis because no recurrences were reported in any of these patients. ${ }^{5,18}$ Third, the follow-up examinations were systemic and comprehensive, which aided in the early detection of asymptomatic recurrences. Finally, a nomogram was built to calculate the recurrence probabilities, which facilitated the selection of high-risk patients. The nomogram showed good calibration, reasonable discrimination, and small "overfitting."

According to the current TNM staging system, the only variable that can be used to subdivide patients with stage Ia NSCLC is T stage (T1a/T1b). ${ }^{2}$ However, the prognostic accuracy of $\mathrm{T}$ stage alone for RFS is not satisfactory $(\mathrm{C}$-index $=0.630)$. The $\mathrm{C}$-index increased to 0.733 when we used all the 5 independent prognostic factors for the prediction of RFS, indicating that these variables were able to add value to the current staging system.

Previous studies have demonstrated the prognostic value of the IASLC/ATS/ERS classification in patients with stage I lung adenocarcinoma. ${ }^{5,18}$ On the basis of the RFS analysis, AIS and MIA were defined as low, lepidic predominant; acinar predominant and papillary predominant were defined as intermediate histologic grade; and solid predominant, micropapillary predominant, and invasive mucinous adenocarcinoma were defined as high histologic grade. ${ }^{5,18}$ Our results were consistent with previous reports. Because large cell carcinoma also showed a high grade of clinical behavior in our study and a previous report, ${ }^{19}$ it was categorized into the "high-grade" group in this study along with solid predominant adenocarcinoma, micropapillary predominant adenocarcinoma, and invasive mucinous adenocarcinoma. The "high-grade" group proved to be an independent predictor of RFS, and tumor histology was the most

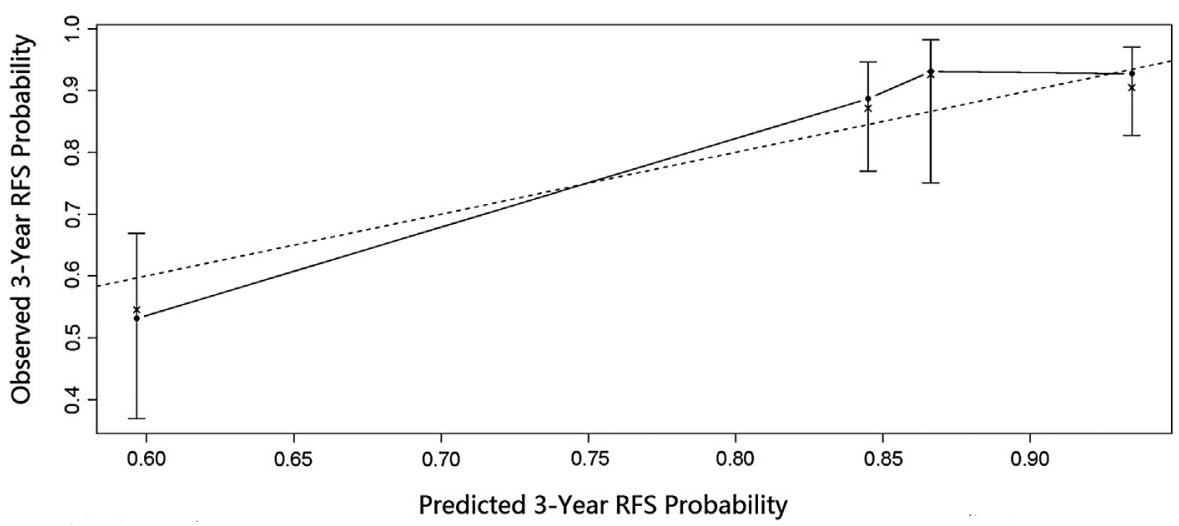

FIGURE 2. Calibration plot of 4 equal contiguous risk ranges showing nomogram-predicted 3-year RFS probabilities with observed probabilities. Dashed line indicates perfect concordance. RFS, Recurrence-free survival. 

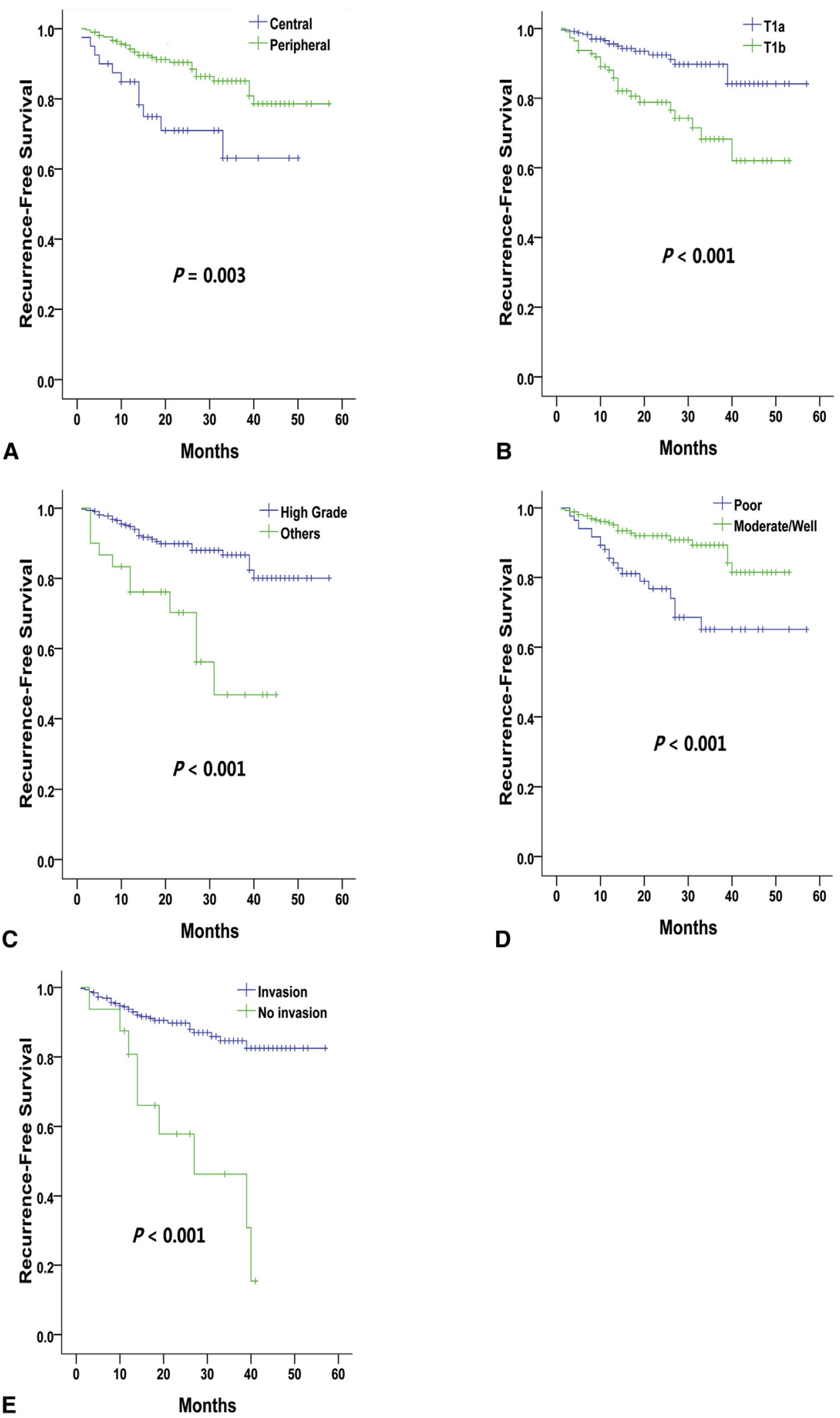

FIGURE 3. Kaplan-Meier survival curves according to tumor location (A), T stage (B), histology (C), differentiation (D), and lymphovascular invasion (E). "High-grade" histology included large cell carcinoma, solid predominant, micropapillary predominant, and invasive mucinous adenocarcinoma. 
determinant variable in the nomogram developed. In addition, the finding that no recurrence occurred in the 25 patients who underwent sublobar resection could be explained by the high proportion $(16 / 25,64 \%)$ of AIS or MIA, and the fact that no "high-grade" histology was present in any of the 25 patients.

Tumor differentiation was considered to provide useful information in defining the aggressiveness of tumors and was reported to be significantly associated with disease recurrence. ${ }^{16}$ Lymphovascular invasion was a step in metastasis. The presence of lymphovascular invasion was a well-recognized unfavorable prognostic factor. ${ }^{3,16}$ Although tumor location was less frequently examined as a possible prognostic factor, Ketchedjian and colleagues ${ }^{20}$ showed that central location was significantly correlated with worse survival in stage I lung adenocarcinoma. This study further demonstrated that central tumor location was an independent poor prognostic factor in stage Ia NSCLC.

The optimal cutoff value of preoperative serum CEA level for predicting the risk of recurrence was identified as $2.5 \mathrm{ng} / \mathrm{mL}$ in a previous study. ${ }^{7}$ We also chose this cutoff point in this study and found an association between CEA level and RFS in univariate analysis. Male sex and smoking history, which both showed a significant association with RFS, were reported to be prognostic factors in other studies. ${ }^{8,21}$ However, the 3 variables were not independent predictors of RFS in our study. Of note, none of these studies included all the clinicopathologic variables in the current study, especially adenocarcinoma subtypes. The survival effects of the 3 variables might be mediated through other factors.

Controversy remains over the benefit of adjuvant chemotherapy for completely resected stage Ia NSCLC. A pooled analysis revealed that the use of cisplatin-based chemotherapy was detrimental for stage Ia NSCLC. ${ }^{22}$ Another meta-analysis showed that adjuvant chemotherapy using tegafur-uracil was effective in stage Ia T1b NSCLC, but not in stage Ia T1a NSCLC. ${ }^{9}$ In addition, Tsuchiya and colleagues $^{3}$ suggested that stage Ia NSCLC with vessel invasion benefited from adjuvant chemotherapy. These results indicated that stage Ia NSCLC with clinicopathologic characteristics associated with recurrence (eg, T1b and vessel invasion) might benefit from postoperative adjuvant chemotherapy. Therefore, we developed a nomogram that is able to calculate the probability of 3-year RFS in stage Ia NSCLC. Physicians can use this nomogram to estimate the individual patient's risk of developing recurrence and choose the appropriate therapeutic strategies, including adjuvant chemotherapy, on the basis of the risk level.

\section{Study Limitations}

The major limitation of our study is that this prediction model is built retrospectively, and its application to guide postoperative management of stage Ia NSCLC has not been demonstrated. For instance, although we can estimate the probability of recurrence using this nomogram, we are not able to decide exactly at what risk threshold adjuvant chemotherapy should be triggered until a prospective study addressing the correlation between the risk of recurrence and the benefits from adjuvant chemotherapy in patients with stage Ia NSCLC is performed. Therefore, both the predictive accuracy of the nomogram and its potential application should be validated in further prospective studies.

Other factors worthy of note are the relatively short follow-up, the small number of events, and the total cases. Although a recent study of resected early-stage NSCLC by Lou and colleagues ${ }^{23}$ reported that the risk of recurrence ranged from $6 \%$ to $10 \%$ per person-year during the first 4 years after surgery, but decreased significantly to $2 \%$ thereafter, we admit that more recurrences will occur in this series of patients given longer follow-up. In addition, the small sample size of this study limits the identification of predictive factors for each type of recurrence (locoregional or distant). Furthermore, the event number is too small to examine the possible interplay of these predictive factors. Future studies with larger samples and longer follow-up are warranted to validate our findings.

This model is built only on Chinese patients, and the proportion of never-smokers in this series of patients is relatively high. Moreover, the majority of tumors are adenocarcinomas. The generalizability of this prediction model needs to be externally validated using databases especially from other countries, considering the ethnic differences in epidemiology and clinical behaviors. ${ }^{24}$

Positron emission tomography (PET)/CT might add value to this prognostic model. ${ }^{17}$ However, because the expense of PET/CT is not covered by insurance in China, only a small proportion of patients in this study received this examination. Although we acknowledge this is a regrettable loss of opportunity, our prediction model can calculate a pretest probability that can be used to obtain a post-test probability after a PET/CT scan according to Bayes' theorem.

Some may criticize that the C-index (0.733) of our prediction model was only fair. Although genetic models may have a higher C-index, the identified survival-related genes usually lack consistency. ${ }^{25}$ Moreover, specific laboratory equipment and expertise are required to acquire reliable expression levels of targeted genes, whereas a clinicopathologic prediction model is more applicable to users. Finally, using Bayes' theorem, our prediction model also can be incorporated into genetic models if necessary.

\section{CONCLUSIONS}

Patients with stage Ia NSCLC are a heterogeneous group with different risks of recurrence. We developed a 5-predictor clinicopathologic prediction model that can stratify 
these patients into different prognostic categories and help to select the appropriate postoperative management.

\section{References}

1. Jemal A, Bray F, Center MM, Ferlay J, Ward E, Forman D. Global cancer statistics. CA Cancer J Clin. 2011;61:69-90.

2. Detterbeck FC, Boffa DJ, Tanoue LT. The new lung cancer staging system. Chest. 2009; 136:260-71

3. Tsuchiya T, Akamine S, Muraoka M, Kamohara R, Tsuji K, Urabe S, et al. Stage IA non-small cell lung cancer: vessel invasion is a poor prognostic factor and a new target of adjuvant chemotherapy. Lung Cancer. 2007;56:341-8.

4. al-Kattan K, Sepsas E, Fountain SW, Townsend ER. Disease recurrence after resection for stage I lung cancer. Eur J Cardiothoracic Surg. 1997;12:380-4.

5. Yoshizawa A, Motoi N, Riely GJ, Sima CS, Gerald WL, Kris MG, et al. Impact of proposed IASLC/ATS/ERS classification of lung adenocarcinoma: prognostic subgroups and implications for further revision of staging based on analysis of 514 stage I cases. Mod Pathol. 2011;24:653-64.

6. Ye C, Masterman JR, Huberman MS, Gangadharan SP, McDonald DC, Kent MS, et al. Subdivision of the T1 size descriptor for stage I non-small cell lung cancer has prognostic value: a single institution experience. Chest. 2009;136:710-5.

7. Kato T, Ishikawa K, Aragaki M, Sato M, Okamoto K, Ishibashi T, et al. Optimal predictive value of preoperative serum carcinoembryonic antigen for surgical outcomes in stage I non-small cell lung cancer: differences according to histology and smoking status. J Surg Oncol. 2013;107:619-24.

8. Wisnivesky JP, Halm EA. Sex differences in lung cancer survival: do tumors behave differently in elderly women? J Clin Oncol. 2007;25:1705-12.

9. Hamada C, Tsuboi M, Ohta M, Fujimura S, Kodama K, Imaizumi M, et al. Effect of postoperative adjuvant chemotherapy with tegafur-uracil on survival in patients with stage IA non-small cell lung cancer: an exploratory analysis from a meta-analysis of six randomized controlled trials. J Clin Oncol. 2009;4:1511-6.

10. Kato H, Ichinose Y, Ohta M, Hata E, Tsubota N, Tada H, et al. A randomized trial of adjuvant chemotherapy with uracil-tegafur for adenocarcinoma of the lung. N Engl J Med. 2004;350:1713-21.

11. Travis WD, Brambilla E, Noguchi M, Nicholson AG, Geisinger KR, Yatabe Y, et al. International Association for the Study of Lung Cancer/American Thoracic Society/European Respiratory Society International Multidisciplinary Classification of Lung Adenocarcinoma. J Thorac Oncol. 2011;6:244-85.
12. Travis WD, Brambilla E, Muller-Hermelink HK. Pathology and Genetics of Tumours of the Lung, Pleura, Thymus, and Heart. Lyon: IARC Press; 2004.

13. Martini N, Melamed MR. Multiple primary lung cancers. J Thorac Cardiovasc Surg. 1975;7:606-12.

14. Harrell FE Jr, Califf RM, Pryor DB, Lee KL, Rosati RA. Evaluating the yield of medical tests. JAMA. 1982;247:2543-6.

15. Harrell FE Jr, Lee KL, Mark DB. Multivariable prognostic models: issues in developing models, evaluating assumptions and adequacy, and measuring and reducing errors. Stat Med. 1996;15:361-87.

16. Shimada Y, Saji H, Yoshida K, Kakihana M, Honda H, Nomura M, et al. Pathological vascular invasion and tumor differentiation predict cancer recurrence in stage IA non-small-cell lung cancer after complete surgical resection. $J$ Thorac Oncol. 2012;7:1263-70.

17. Shiono S, Abiko M, Sato T. Positron emission tomography/computed tomography and lymphovascular invasion predict recurrence in stage I lung cancers J Thorac Oncol. 2011;6:43-7.

18. Woo T, Okudela K, Mitsui H, Tajiri M, Yamamoto T, Rino Y, et al. Prognostic value of the IASLC/ATS/ERS classification of lung adenocarcinoma in stage I disease of Japanese cases. Pathol Int. 2012;62:785-91.

19. Downey RS, Sewell CW, Mansour KA. Large cell carcinoma of the lung: a highly aggressive tumor with dismal prognosis. Ann Thorac Surg. 1989;47:806-8.

20. Ketchedjian A, Daly BD, Fernando HC, Florin L, Hunter CJ, Morelli DM, et al. Location as an important predictor of lymph node involvement for pulmonary adenocarcinoma. J Thorac Cardiovasc Surg. 2006;132:544-8.

21. Tammemagi CM, Neslund-Dudas C, Simoff M, Kvale P. Smoking and lung cancer survival: the role of comorbidity and treatment. Chest. 2004;125:27-37.

22. Pignon JP, Tribodet H, Scagliotti GV, Douillard JY, Shepherd FA, Stephens RJ et al. Lung adjuvant cisplatin evaluation: a pooled analysis by the LACE Collaborative Group. J Clin Oncol. 2008;26:3552-9.

23. Lou F, Huang J, Sima CS, Dycoco J, Rusch V, Bach PB. Patterns of recurrence and second primary lung cancer in early-stage lung cancer survivors followed with routine computed tomography surveillance. J Thorac Cardiovasc Surg. 2013;145:75-82.

24. Zhou W, Christiani DC. East meets West: ethnic differences in epidemiology and clinical behaviors of lung cancer between East Asians and Caucasians. Chin J Cancer. 2011;30:287-92.

25. Lu Y, Wang L, Liu P, Yang P, You M. Gene-expression signature predicts postoperative recurrence in stage I non-small cell lung cancer patients. PLOS One. 2012;7:e30880. 